\title{
DIPOLE DERIVATIVES AND INFRARED INTENSITIES OF THE ESTER GROUP. AN AB INITIO AND FORCE FIELD STUDY OF METHYL ACETATE
}

\author{
J. DYBAL* and S. KRIMM \\ Biophysics Research Division and Department of Physics, University of Michigan, Ann Arbor, \\ MI 48109 (U.S.A.)
}

(Received 6 January 1988)

\begin{abstract}
Dipole derivatives have been obtained (using both 3-21G and 6-31G basis sets) for methyl acetate. Using a force field refined from experimental data on methyl acetate and its deuterated derivatives, infrared intensities have been calculated. The good frequency and intensity agreement indicate that these force constants and dipole derivatives can serve as satisfactory transferable parameters for the ester group.
\end{abstract}

\section{INTRODUCTION}

Dipole derivatives provide important insights into interactions between similar groups on adjacent molecules, which determine band splittings in vibrational spectra, and permit calculation of infrared (IR) intensities, which can serve as an independent test of force fields. Such ab initio derivatives for the peptide group $[1,2]$ have validated the proposal that transition dipole coupling accounts for amide mode band splittings in spectra of polypeptides $[3,4]$, and the corresponding IR intensities have provided support for our empirically refined force field for polyglycine I [2].

This paper presents dipole derivatives and IR intensities for the ester group. We have used methyl acetate (MA) as a model, and have tried to derive a set of transferable parameters that will yield reliable frequencies and IR intensities for this group. In the process, we have refined an alternative intramolecular valence force field for this molecule. The transferability of the force field and group moment derivatives will be tested in future work on poly (methyl methacrylate) and polydepsipeptides.

Infrared and Raman spectra have been reported for MA [5, 6] and its deu-

\footnotetext{
*Present address: Institute of Macromolecular Chemistry, Czechoslovak Academy of Sciences, 16206 Prague 6, Czechoslovakia.
} 
terated derivatives [6], and tentative band assignments have been given. Force field refinements for MA have been done in a methyl-group point-mass approximation [7], as part of a transferable force field for a series of molecules containing $\mathrm{C}=\mathrm{O}$ groups [8], and on the individual molecule [9]. Our force field refinement was based only on experimental vibrational data for MA and its deuterated derivatives. The analysis of IR band intensities followed previous procedures [2], calculating ab initio dipole moment derivatives with respect to local symmetry coordinates. Intensities were determined using both our own and the Hollenstein and Günthard [8] force fields. Comparison of the results with experiments shows that the observed IR intensities can be very well reproduced.

\section{CALCULATIONS}

In the normal mode and ab initio calculations, we used the equilibrium geometrical structure of the MA molecule obtained by a Hartree-Fock optimization with the 4-21G basis set [10]. The local symmetry internal coordinates $\overrightarrow{\mathbf{S}}$, constructed from the internal coordinates defined in the standard way, are listed in Table 1 with the numbering of atoms shown in Fig. 1. The out-ofplane angle bends, $\Delta \omega$, and the torsions, $\Delta \tau$, are defined as before [11]. The vibrational band frequencies of $\mathrm{CH}_{3} \mathrm{COOCH}_{3}, \mathrm{CH}_{3} \mathrm{COOCD}_{3}, \mathrm{CD}_{3} \mathrm{COOCH}_{3}$, and $\mathrm{CD}_{3} \mathrm{COOCD}_{3}[6]$ were used in the refinement of the intramolecular valence force field. Since the force field is intended to be transferred to polymers, it was refined with respect to band frequencies measured in the spectra of MA in the condensed phase.

A combined valence force field of acetic acid [12] and of methyl formate [13] was used to derive an initial set of MA force constants. The refinement was started with a diagonal force field, since we wished to keep the number of parameters of the force field to a minimum. The most relevant interaction force constants were included in the force field in the next steps of the refinement, but in such a way that the least-squares method could be applied to the entire force field in one run. Calculations showed that some changes in the proposed band assignments [6] were necessary, most of the alterations having already been proposed [8]. The fit between observed and calculated frequencies was improved iteratively until the sum of the squared residuals became stationary.

Dipole moments of MA were calculated by the ab initio Hartree-Fock method. We used a version of the Gaussian 76 program system [14] that was modified to run on a VAX computer [2]. The dipole moments were computed using both the $3-21 \mathrm{G}$ and the $6-31 \mathrm{G}$ basis sets. The dipole moment derivatives, $\partial \vec{\mu} / \partial \mathrm{S}_{\mathrm{i}}$, with respect to the local symmetry coordinates $\vec{S}$ were evaluated by numerical differentiation. Bonds were distorted by $\pm 0.01 \AA$ and angles by $\pm 0.025 \mathrm{rad}$. 


\section{TABLE 1}

Local symmetry coordinates of methyl acetate

\begin{tabular}{|c|c|c|}
\hline Species & Symmetry coordinate & Description \\
\hline$A^{\prime}$ & $\begin{array}{l}S_{1}=\Delta\left(2 r_{49}-r_{4,10}-r_{4,11}\right) / \sqrt{6} \\
S_{2}=\Delta\left(2 r_{56}-r_{58}-r_{57}\right) / \sqrt{6} \\
S_{3}=\Delta\left(r_{56}+r_{57}+r_{58}\right) / \sqrt{3} \\
S_{4}=\Delta\left(r_{49}+r_{4,10}+r_{4,11}\right) / \sqrt{3} \\
S_{5}=\Delta r_{23} \\
S_{6}=\Delta\left(2 \alpha_{758}-\alpha_{658}-\alpha_{657}\right) / \sqrt{6} \\
S_{7}=\Delta\left(2 \alpha_{10,4,11}-\alpha_{94,11}-\alpha_{94,10}\right) / \sqrt{6} \\
S_{8}=\Delta\left(\alpha_{657}+\alpha_{658}+\alpha_{758}-\alpha_{156}-\alpha_{157}-\alpha_{158}\right) / \sqrt{6} \\
S_{9}=\Delta\left(\alpha_{94,10}+\alpha_{94,11}+\alpha_{10,4,11}-\alpha_{249}-\alpha_{24,10}-\alpha_{24,11}\right) / \sqrt{6} \\
S_{10}=\Delta r_{12} \\
S_{11}=\Delta\left(2 \alpha_{156}-\alpha_{157}-\alpha_{158}\right) / \sqrt{6} \\
S_{12}=\Delta r_{24} \\
S_{13}=\Delta\left(2 \alpha_{249}-\alpha_{24,10}-\alpha_{24,11}\right) / \sqrt{6} \\
S_{14}=\Delta r_{15} \\
S_{15}=\Delta\left(\alpha_{324}-\alpha_{123}\right) / \sqrt{2} \\
S_{16}=\Delta\left(2 \alpha_{124}-\alpha_{324}-\alpha_{123}\right) / \sqrt{6} \\
S_{17}=\Delta \alpha_{215}\end{array}$ & 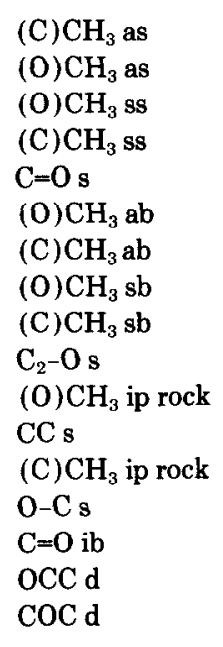 \\
\hline$A^{\prime \prime}$ & $\begin{array}{l}S_{18}=\Delta\left(r_{4,10}-r_{4,11}\right) / \sqrt{2} \\
S_{19}=\Delta\left(r_{57}-r_{58}\right) / \sqrt{2} \\
S_{20}=\Delta\left(\alpha_{658}-\alpha_{657}\right) / \sqrt{2} \\
S_{21}=\Delta\left(\alpha_{94,11}-\alpha_{94,10}\right) / \sqrt{2} \\
S_{22}=\Delta\left(\alpha_{157}-\alpha_{158}\right) / \sqrt{2} \\
S_{23}=\Delta\left(\alpha_{24,10}-\alpha_{24,11}\right) / \sqrt{2} \\
S_{24}=\Delta \omega \\
S_{25}=\Delta\left(\tau_{3215}+\tau_{4215}\right) / \sqrt{2} \\
S_{26}=\Delta\left(\tau_{2156}+\tau_{2157}+\tau_{2158}\right) / \sqrt{3} \\
S_{27}=\Delta\left(\tau_{1249}+\tau_{124,10}+\tau_{124,11}+\tau_{3249}+\tau_{324,10}+\tau_{324,11}\right) / \sqrt{6}\end{array}$ & $\begin{array}{l}\text { (C) } \mathrm{CH}_{3} \text { as } \\
\text { (O) } \mathrm{CH}_{3} \text { as } \\
(\mathrm{O}) \mathrm{CH}_{3} \text { ab } \\
(\mathrm{C}) \mathrm{CH}_{3} \text { ab } \\
\text { (O) } \mathrm{CH}_{3} \text { op rock } \\
\text { (C) } \mathrm{CH}_{3} \text { op rock } \\
\mathrm{C}=\mathrm{O} \text { ob } \\
\mathrm{C}_{2}-\mathrm{O} \text { tor } \\
\text { O-C tor } \\
\mathrm{CC} \text { tor }\end{array}$ \\
\hline
\end{tabular}

${ }^{\mathrm{a}_{\mathrm{S}}}=$ stretch, as = antisymmetric stretch, $\mathrm{ss}=$ symmetric stretch, $\mathrm{ab}=$ antisymmetric bend, $\mathrm{sb}=$ symmetric bend, $\mathrm{ib}=$ in-plane bend, $\mathrm{ob}=$ out-of-plane bend, $\mathrm{op}=$ out-of-plane, $\mathrm{d}=$ deformation, tor $=$ torsion.

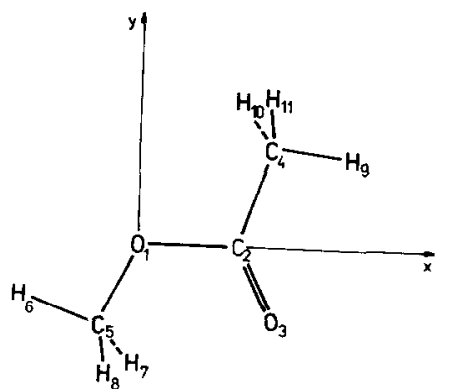

Fig. 1. Methyl acetate molecule, with coordinate system and atom numbering. 
Cartesian displacements corresponding to each internal coordinate distortion were obtained from $\overrightarrow{\mathbf{X}}=\mathbf{M}^{-1} \mathbf{B}\left(\mathbf{B} \mathbf{M}^{-1}\right)^{-1} \overrightarrow{\mathbf{S}}$, where $\mathbf{M}$ is the matrix of atomic masses and $\overrightarrow{\mathbf{S}}=\mathbf{B} \overrightarrow{\mathbf{X}}$. The transformation of the dipole moment derivatives into the normal coordinate basis $\overrightarrow{\mathbf{Q}}$ was performed using the relation

$\frac{\partial \overrightarrow{\boldsymbol{\mu}}}{\partial Q_{\alpha}}=\sum_{i} \mathbf{L}_{i \alpha} \frac{\partial \vec{\mu}}{\partial S_{i}}$

The eigenvector matrix $\mathbf{L}$, defined by $\overrightarrow{\mathbf{S}}=\mathbf{L} \overrightarrow{\mathbf{Q}}$, was obtained from the normal mode calculations. The integrated infrared intensity of the $\alpha$ th mode is then given by

$A_{\alpha}=\frac{N \pi}{3 c^{2}}\left(\frac{\partial \vec{\mu}}{\partial Q_{\alpha}}\right)^{2}$

where $N$ is Avogadro's number and $c$ is the velocity of light.

RESULTS AND DISCUSSION

Our final set of valence force constants for MA is given in Table 2. The force field was refined in the non-redundant local symmetry coordinates of Table 1.

TABLE 2

Valence force constants for methyl acetate

\begin{tabular}{rlrlll}
\hline No. & Coordinates & Value & No. & Coordinates & Value \\
\hline 1 & 1,1 & $4.852(0.029)$ & 19 & 19,19 & $4.789(0.029)$ \\
2 & 2,2 & $4.859(0.029)$ & 20 & 20,20 & $0.518(0.006)$ \\
3 & 3,3 & $5.050(0.029)$ & 21 & 21,21 & $0.517(0.006)$ \\
4 & 4,4 & $5.009(0.029)$ & 22 & 22,22 & $0.835(0.012)$ \\
5 & 5,5 & $11.009(0.109)$ & 23 & 23,23 & $0.567(0.026)$ \\
6 & 6,6 & $0.485(0.007)$ & 24 & 24,24 & $0.734(0.038)$ \\
7 & 7,7 & $0.507(0.007)$ & 25 & 25,25 & $0.210(0.023)$ \\
8 & 8,8 & $0.661(0.009)$ & 26 & 26,26 & 0.032 \\
9 & 9,9 & $0.563(0.009)$ & 27 & 27,27 & 0.024 \\
10 & 10,10 & $5.031(0.223)$ & 28 & 5,10 & $0.971(0.055)$ \\
11 & 11,11 & $0.868(0.021)$ & 29 & 5,12 & 0.971 \\
12 & 12,12 & $4.046(0.176)$ & 30 & 8,14 & $-0.639(0.050)$ \\
13 & 13,13 & $0.685(0.019)$ & 31 & 9,12 & $-0.537(0.022)$ \\
14 & 14,14 & $5.062(0.132)$ & 32 & 9,16 & $-0.162(0.042)$ \\
15 & 15,15 & $1.259(0.042)$ & 33 & 10,14 & $0.731(0.222)$ \\
16 & 16,16 & 1.129 & 34 & 10,15 & $-0.219(0.023)$ \\
17 & 17,17 & $1.729(0.133)$ & 35 & 12,15 & 0.219 \\
18 & 18,18 & $4.785(0.029)$ & 36 & 23,24 & $-0.112(0.014)$ \\
\hline
\end{tabular}

${ }^{a}$ Numbers in parentheses are dispersions for refined force constants. 
TABLE 3

Observed and calculated frequencies (in $\mathrm{cm}^{-1}$ ) of methyl acetate

\begin{tabular}{|c|c|c|c|c|}
\hline & Obs. & Calc. & No. & Potential energy distribution ${ }^{a}$ \\
\hline \multicolumn{5}{|c|}{$\mathrm{CH}_{3} \mathrm{COOCH}_{3}$} \\
\hline \multirow[t]{17}{*}{$A^{\prime}$} & 3028 & 3030 & 1 & $(0) \mathrm{CH}_{3}$ as (99) \\
\hline & 3028 & 3023 & 2 & (C) $\mathrm{CH}_{3}$ as (99) \\
\hline & 2955 & 2956 & 3 & (0) $\mathrm{CH}_{3} \mathrm{ss}(100)$ \\
\hline & 2940 & 2947 & 4 & (C) $\mathrm{CH}_{3} \mathrm{ss}(100)$ \\
\hline & 1747 & 1747 & 5 & $\mathrm{C}=\mathrm{Os}(83), \mathrm{OCC} \mathrm{d}(10)$ \\
\hline & 1455 & 1458 & 6 & $(\mathrm{O}) \mathrm{CH}_{3} \mathrm{ab}(75),(\mathrm{O}) \mathrm{CH}_{3} \mathrm{r}(23)$ \\
\hline & 1438 & 1440 & 7 & (C) $\mathrm{CH}_{3} \mathrm{ab}(88)$ \\
\hline & 1438 & 1433 & 8 & (0) $\mathrm{CH}_{3} \mathrm{sb}(99)$ \\
\hline & 1372 & 1374 & 9 & (C) $\mathrm{CH}_{3} \mathrm{sb}(68)$ \\
\hline & 1249 & 1251 & 10 & (C) $\mathrm{CH}_{3} \mathrm{sb}(31), \mathrm{CC} s(25), \mathrm{C}_{2}-\mathrm{Os}(23), \mathrm{C}=\mathrm{O} \mathrm{ib}(19)$ \\
\hline & 1190 & 1195 & 11 & $(0) \mathrm{CH}_{3} \mathrm{r}(56),(0) \mathrm{CH}_{3} \mathrm{ab}(18), \mathrm{COC} \mathrm{d}(11)$ \\
\hline & 1051 & 1054 & 12 & $\mathrm{O}-\mathrm{Cs}(66),(\mathrm{C}) \mathrm{CH}_{3} \mathrm{r}(18)$ \\
\hline & 982 & 998 & 13 & (C) $\mathrm{CH}_{3} \mathrm{r}(56), \mathrm{O}-\mathrm{C} \mathrm{s}(20), \mathrm{CC} \mathrm{s}(13)$ \\
\hline & 846 & 852 & 14 & $\mathrm{C}_{2}-\mathrm{Os}(37), \mathrm{CC} \mathrm{s}(22), \mathrm{COC} \mathrm{d}(10)$ \\
\hline & 638 & 633 & 15 & $\mathrm{C}=\mathrm{O} \mathrm{ib}(43), \mathrm{CC} s(34), \mathrm{COC} \mathrm{d}(10)$ \\
\hline & 433 & 434 & 16 & OCC d(46), COC d (14), $\mathrm{C}_{2}-\mathrm{Os}(13), \mathrm{C}=\mathrm{O} \mathrm{ib}(12)$ \\
\hline & 302 & 299 & 17 & $\operatorname{COC~d}(47), 0 C C \mathrm{~d}(39), \mathrm{C}=\mathrm{O} \mathrm{ib}(10)$ \\
\hline \multirow[t]{10}{*}{$A^{\prime \prime}$} & 3002 & 3001 & 18 & $(\mathrm{O}) \mathrm{CH}_{3}$ as (99) \\
\hline & 3002 & 3000 & 19 & (C) $\mathrm{CH}_{3}$ as (99) \\
\hline & 1462 & 1467 & 20 & (O) $\mathrm{CH}_{3} \mathrm{ab}(88),(\mathrm{O}) \mathrm{CH}_{3} \mathrm{r}(12)$ \\
\hline & 1445 & 1444 & 21 & (C) $\mathrm{CH}_{3}$ ab (94) \\
\hline & 1161 & 1163 & 22 & $(\mathrm{O}) \mathrm{CH}_{3} \mathrm{r}(85),(\mathrm{O}) \mathrm{CH}_{3} \mathrm{ab}(11)$ \\
\hline & 1051 & 1060 & 23 & (C) $\mathrm{CH}_{3} \mathrm{r}(64), \mathrm{C}=\mathrm{O} \mathrm{ob}(17)$ \\
\hline & 609 & 615 & 24 & $\mathrm{C}=\mathrm{O} \mathrm{ob}(66),(\mathrm{C}) \mathrm{CH}_{3} \mathrm{r}(26)$ \\
\hline & 203 & 211 & 25 & $\mathrm{C}_{2}-\mathrm{O}$ tor $(80), \mathrm{O}-\mathrm{C}$ tor $(10)$ \\
\hline & - & 131 & 26 & $\mathrm{O}-\mathrm{C}$ tor $(85), \mathrm{C}_{2}-\mathrm{O}$ tor $(12)$ \\
\hline & - & 116 & 27 & $\mathrm{CC}$ tor $(96)$ \\
\hline \multicolumn{5}{|c|}{$\mathrm{CH}_{3} \mathrm{COOCD}_{3}$} \\
\hline$A^{\prime}$ & 3023 & 3023 & & (C) $\mathrm{CH}_{3}$ as (99) \\
\hline & 2950 & 2947 & & (C) $\mathrm{CH}_{3} \mathrm{ss}(100)$ \\
\hline & 2259 & 2267 & & $(0) \mathrm{CD}_{3}$ as $(97)$ \\
\hline & 2085 & 2124 & & $(0) \mathrm{CD}_{3} \mathrm{ss}(98)$ \\
\hline & 1746 & 1745 & & $\mathrm{C}=\mathrm{Os}(83), \mathrm{OCC} \mathrm{d}(10)$ \\
\hline & 1438 & 1438 & & (C) $\mathrm{CH}_{3} \mathrm{ab}(90)$ \\
\hline & 1372 & 1377 & & (C) $\mathrm{CH}_{3} \mathrm{sb}(65), \mathrm{C}_{2}-\mathrm{Os}(12), \mathrm{C}=\mathrm{O} \mathrm{ib}(10)$ \\
\hline & 1265 & 1259 & & (C) $\mathrm{CH}_{3} \mathrm{sb}(33), \mathrm{CC} \mathrm{s}(24), \mathrm{C}_{2}-\mathrm{Os}(23), \mathrm{C}=\mathrm{O} \mathrm{ib}(18)$ \\
\hline & 1093 & 1096 & & $\mathrm{O}-\mathrm{Cs}(44),(\mathrm{O}) \mathrm{CD}_{3} \mathrm{sb}(43)$ \\
\hline & 1065 & 1061 & & $(0) \mathrm{CD}_{3} \mathrm{sb}(65),(0) \mathrm{CD}_{3} \mathrm{r}(13)$ \\
\hline & 1038 & 1039 & & (O) $\mathrm{CD}_{3} \mathrm{ab}(69),(0) \mathrm{CD}_{3} \mathrm{r}(19)$ \\
\hline & 1017 & 1008 & & (C) $\mathrm{CH}_{3} \mathrm{r}(73), \mathrm{C}_{2}-\mathrm{Os}(10)$ \\
\hline & 957 & 956 & & $\mathrm{O}-\mathrm{C} \mathrm{s}(42), \mathrm{CC} \mathrm{s}(21),(0) \mathrm{CD}_{3} \mathrm{r}(15)$ \\
\hline & 778 & 778 & & $(\mathrm{O}) \mathrm{CD}_{3} \mathrm{r}(37), \mathrm{C}_{2}-\mathrm{Os}(24), \mathrm{CCs}(20)$ \\
\hline & 616 & 617 & & $\mathrm{C}=\mathrm{O} \mathrm{ib}(46), \mathrm{CC}_{\mathrm{s}}(26)$ \\
\hline & 419 & 422 & & $\mathrm{OCC} d(50), \mathrm{C}_{2}-\mathrm{Os}(12), \mathrm{CC} \mathrm{s}(12), \operatorname{COC} \mathrm{d}(12)$ \\
\hline & 278 & 277 & & $\operatorname{COCd}(51), 0 C C d(33), C=O \mathrm{ib}(10)$ \\
\hline
\end{tabular}


TABLE 3 (continued)

\begin{tabular}{|c|c|c|c|c|}
\hline & Obs. & Calc. & No. & Potential energy distribution ${ }^{a}$ \\
\hline \multirow[t]{10}{*}{$A^{\prime \prime}$} & 2992 & 3000 & & (C) $\mathrm{CH}_{3}$ as (99) \\
\hline & 2277 & 2238 & & (0) $\mathrm{CD}_{3}$ as $(98)$ \\
\hline & 1446 & 1444 & & (C) $\mathrm{CH}_{3} \mathrm{ab}(94)$ \\
\hline & 1065 & 1064 & & (C) $\mathrm{CH}_{3} \mathrm{r}(62), \mathrm{C}=\mathrm{O}$ ob(18) \\
\hline & 1055 & 1051 & & (O) $\mathrm{CD}_{3} \mathrm{ab}(93)$ \\
\hline & 900 & 900 & & $(0) \mathrm{CD}_{3} \mathrm{r}(90)$ \\
\hline & 605 & 611 & & $\mathrm{C}=\mathrm{O} a b(68),(\mathrm{C}) \mathrm{CH}_{3} \mathrm{r}(27)$ \\
\hline & 200 & 196 & & $\mathrm{C}_{2}-\mathrm{O}$ tor $(82)$ \\
\hline & - & 117 & & $\mathrm{CC}$ tor $(96)$ \\
\hline & - & 95 & & $\mathrm{O}-\mathrm{C}$ tor $(90)$ \\
\hline \multicolumn{5}{|c|}{$\mathrm{CD}_{3} \mathrm{COOCH}_{3}$} \\
\hline \multirow[t]{17}{*}{$A^{\prime}$} & 3030 & 3030 & & $(0) \mathrm{CH}_{3}$ as $(99)$ \\
\hline & 2956 & 2956 & & $(0) \mathrm{CH}_{3} \mathrm{ss}(100)$ \\
\hline & 2250 & 2259 & & (C) $\mathrm{CD}_{3}$ as $(97)$ \\
\hline & 2119 & 2119 & & (C) $\mathrm{CD}_{3} \mathrm{ss}(99)$ \\
\hline & 1742 & 1741 & & $\mathrm{C}=\mathrm{Os}(84)$ \\
\hline & 1453 & 1458 & & $(\mathrm{O}) \mathrm{CH}_{3} \mathrm{ab}(76),(\mathrm{O}) \mathrm{CH}_{3} \mathrm{r}(23)$ \\
\hline & 1435 & 1434 & & $(\mathrm{O}) \mathrm{CH}_{3} \mathrm{sb}(100)$ \\
\hline & 1263 & 1276 & & $\mathrm{C}_{2}-\mathrm{Os}(41), \mathrm{C}=\mathrm{O} \mathrm{ib}(30), \mathrm{CC} \mathrm{s}(22)$ \\
\hline & 1190 & 1195 & & $(\mathrm{O}) \mathrm{CH}_{3} \mathrm{r}(52),(\mathrm{O}) \mathrm{CH}_{3} \mathrm{ab}(16), \mathrm{COC} \mathrm{d}(11)$ \\
\hline & 1080 & 1066 & & $\mathrm{O}-\mathrm{Cs}(53),(\mathrm{C}) \mathrm{CD}_{3} \mathrm{sb}(19),(\mathrm{O}) \mathrm{CH}_{3} \mathrm{r}(11)$ \\
\hline & 1032 & 1033 & & (C) $\mathrm{CD}_{3} \mathrm{ab}(84), \mathrm{O}-\mathrm{Cs}(10)$ \\
\hline & 995 & 997 & & (C) $\mathrm{CD}_{3} \mathrm{sb}(62), \mathrm{CC} \mathrm{s}(19), \mathrm{O}-\mathrm{Cs}(17)$ \\
\hline & 864 & 847 & & $\mathrm{C}_{2}-\mathrm{Os}(20), \mathrm{CC} \mathrm{s}(14),(\mathrm{C}) \mathrm{CD}_{3} \mathrm{r}(10)$ \\
\hline & 780 & 781 & & (C) $\mathrm{CD}_{3} \mathrm{r}(66), \mathrm{C}_{2}-\mathrm{Os}(18)$ \\
\hline & 596 & 594 & & $\mathrm{C}=0 \mathrm{ib}(36), \mathrm{CC} \mathrm{s}(35)$ \\
\hline & 401 & 411 & & $\operatorname{OCC~d}(34), \operatorname{COCd}(20), \mathrm{C}=\mathrm{O} \mathrm{ib}(17), \mathrm{C}_{2}-\mathrm{Os}(13)$ \\
\hline & 290 & 289 & & $\operatorname{OCC} d(47), \operatorname{COC} d(41)$ \\
\hline \multirow[t]{10}{*}{$A^{\prime \prime}$} & 2998 & 3001 & & $(\mathrm{O}) \mathrm{CH}_{3}$ as $(99)$ \\
\hline & 2250 & 2238 & & (C) $\mathrm{CD}_{3}$ as $(98)$ \\
\hline & 1460 & 1467 & & $(\mathrm{O}) \mathrm{CH}_{3}$ ab(88), (O) $\mathrm{CH}_{3} \mathrm{r}(12)$ \\
\hline & 1158 & 1162 & & $(0) \mathrm{CH}_{3} \mathrm{r}(86),(\mathrm{O}) \mathrm{CH}_{3} \mathrm{ab}(11)$ \\
\hline & 1032 & 1039 & & (C) $\mathrm{CD}_{3} \mathrm{ab}(96)$ \\
\hline & 918 & 920 & & (C) $\mathrm{CD}_{3} \mathrm{r}(43), \mathrm{C}=\mathrm{O}$ ob (39) \\
\hline & 529 & 532 & & (C) $\mathrm{CD}_{3} \mathrm{r}(46), \mathrm{C}=\mathrm{O}$ ob (45) \\
\hline & 208 & 204 & & $\mathrm{C}_{2}-\mathrm{O}$ tor $(76), \mathrm{O}-\mathrm{C}$ tor $(11)$ \\
\hline & - & 130 & & $\mathrm{O}-\mathrm{C}$ tor $(87), \mathrm{C}_{2}-\mathrm{O}$ tor $(13)$ \\
\hline & - & 85 & & $\mathrm{CC}$ tor $(99)$ \\
\hline \multicolumn{5}{|c|}{$\mathrm{CD}_{3} \mathrm{COOCD}_{3}$} \\
\hline \multirow[t]{6}{*}{$A^{\prime}$} & 2258 & 2267 & & $(0) \mathrm{CD}_{3}$ as $(97)$ \\
\hline & 2258 & 2259 & & (C) $\mathrm{CD}_{3}$ as $(96)$ \\
\hline & 2125 & 2124 & & $(0) \mathrm{CD}_{3} \mathrm{ss}(98)$ \\
\hline & 2125 & 2119 & & (C) $\mathrm{CD}_{3} \mathrm{ss}(99)$ \\
\hline & 1738 & 1740 & & $\mathrm{C}=\mathrm{O} s(84)$ \\
\hline & 1283 & 1286 & & $\mathrm{C}_{2}-\mathrm{Os}(43), \mathrm{C}=\mathrm{O} \mathrm{ib}(31), \mathrm{CC} \mathrm{s}(24)$ \\
\hline
\end{tabular}


TABLE 3 (continued)

\begin{tabular}{|c|c|c|c|c|}
\hline & Obs. & Calc. & No. & Potential energy distribution ${ }^{a}$ \\
\hline & 1096 & 1105 & & $\mathrm{O}-\mathrm{Cs}(52),(0) \mathrm{CD}_{3} \mathrm{sb}(21), \mathrm{COCd}(11),(0) \mathrm{CD}_{3} \mathrm{r}(10)$ \\
\hline & 1061 & 1066 & & $(0) \mathrm{CD}_{3} \mathrm{sb}(85)$ \\
\hline & 1035 & 1039 & & $(0) \mathrm{CD}_{3} \mathrm{ab}(79),(\mathrm{O}) \mathrm{CD}_{3} \mathrm{r}(13)$ \\
\hline & 1035 & 1032 & & (C) $\mathrm{CD}_{3} \mathrm{ab}(86)$ \\
\hline & 992 & 996 & & (C) $\mathrm{CD}_{3} \mathrm{sb}(87), \mathrm{CC} \mathrm{s}(16)$ \\
\hline & 968 & 959 & & $\mathrm{O}-\mathrm{Cs}(42),(\mathrm{O}) \mathrm{CD}_{3} \mathrm{r}(20), \mathrm{CC} \mathrm{s}(13),(\mathrm{O}) \mathrm{CD}_{3} \mathrm{ab}(10)$ \\
\hline & 829 & 814 & & $(\mathrm{C}) \mathrm{CD}_{3} \mathrm{r}(47), \mathrm{CCs}(11)$ \\
\hline & 745 & 740 & & $(0) \mathrm{CD}_{3} \mathrm{r}(29),(\mathrm{C}) \mathrm{CD}_{3} \mathrm{r}(29), \mathrm{C}_{2}-\mathrm{O} \mathbf{s}(28)$ \\
\hline & 586 & 583 & & $\mathrm{C}=\mathrm{O} \mathrm{ib}(41), \mathrm{CC} s(30)$ \\
\hline & 387 & 397 & & $\operatorname{OCC~d(40),~COC~d(17),~C=O~ib~(12),~} \mathrm{C}_{2}-\mathrm{O} \mathrm{s}(11)$ \\
\hline & 270 & 269 & & $\operatorname{COCd}(46), \operatorname{oCCd}(41)$ \\
\hline \multirow[t]{10}{*}{$A^{\prime \prime}$} & 2270 & 2239 & & $(0) \mathrm{CD}_{3}$ as $(98)$ \\
\hline & 2270 & 2238 & & (C) $\mathrm{CD}_{3}$ as $(98)$ \\
\hline & 1053 & 1052 & & $(0) \mathrm{CD}_{3} \mathrm{ab}(94)$ \\
\hline & 1035 & 1039 & & (C) $\mathrm{CD}_{3} \mathrm{ab}(96)$ \\
\hline & 922 & 936 & & $\mathrm{C}=\mathrm{O}$ ob $(31),(\mathrm{C}) \mathrm{CD}_{3} \mathrm{r}(27),(\mathrm{O}) \mathrm{CD}_{3} \mathrm{r}(25)$ \\
\hline & 896 & 884 & & $(0) \mathrm{CD}_{3} \mathrm{r}(66),(\mathrm{C}) \mathrm{CD}_{3} \mathrm{r}(16)$ \\
\hline & 527 & 529 & & (C) $\mathrm{CD}_{3} \mathrm{r}(46), \mathrm{C}=\mathrm{O}$ ob (46) \\
\hline & 200 & 188 & & $\mathrm{C}_{2}-\mathrm{O}$ tor $(80)$ \\
\hline & - & 97 & & $\mathrm{O}-\mathrm{C}$ tor $(80), \mathrm{CC}$ tor $(10)$ \\
\hline & - & 83 & & $\mathrm{CC}$ tor $(89), 0-\mathrm{C}$ tor $(11)$ \\
\hline
\end{tabular}

${ }^{a}$ Potential energy distribution, diagonal elements $\geqslant 10 \%$. Abbreviations: $\mathrm{s}=$ stretch, as $=$ antisymmetric stretch, $\mathrm{ss}=$ symmetric stretch, $\mathrm{ab}=$ antisymmetric bend, $\mathrm{sb}=$ symmetric bend, ib $=$ in-plane bend, $o b=$ out-of-plane bend, $\mathrm{d}=$ deformation, $\mathrm{r}=$ rock, tor $=$ torsion .

The force constants for methyl torsions $\left(\mathrm{O}-\mathrm{CH}_{3}\right.$ and $\left.\mathrm{C}-\mathrm{CH}_{3}\right)$ were kept fixed at the values transferred from methyl formate [13] and acetic acid [12], because corresponding bands were not observed in the spectra of MA [5, 6]. Force constant No. 16 (OCC deformation) was not refined from its initial value, because it showed very large uncertainties in the course of least-squares fitting of the calculated frequencies. For the same reason, only one parameter was used for the pairs of correlated force constants 28,29 and 34,35 in the refinement of the force field. Since the $\mathrm{CD}_{3}$ stretching region $\left(2100-2300 \mathrm{~cm}^{-1}\right)$ is very complex in the recorded spectra of MA [6] (due to effects such as Fermi resonance), the methyl stretch force constants were fitted only to the band frequencies corresponding to the vibrations of the undeuterated methyl groups.

As can be seen from Table 3, most of the discrepancies between the observed and calculated frequencies are less than $10 \mathrm{~cm}^{-1}$ for all four isotopic species. Several larger differences probably arise from the anharmonicities not included in the calculations. The normal mode calculations indicate that the bands in the $2250-2260 \mathrm{~cm}^{-1}$ region correspond to the $\mathrm{CD}_{3}$ antisymmetric stretch modes in the $A^{\prime}$ block (modes Nos. 1 and 2). Changes in the assignments given in [6] are proposed also for some bands in the $900-1100 \mathrm{~cm}^{-1}$ region of the spectra of $\mathrm{CH}_{3} \mathrm{COOCD}_{3}$ and $\mathrm{CD}_{3} \mathrm{COOCD}_{3}$ (see Table 3).

Dipole moment derivatives, $\partial \vec{\mu} / \partial S_{i}$, were calculated using the $3-21 \mathrm{G}$ and 6 - 
TABLE 4

Dipole moment derivatives (in D/A or D/rad) of methyl acetate (6-31G basis)

\begin{tabular}{|c|c|c|c|c|c|}
\hline Coordinate $^{a}$ & $\frac{\partial \mu_{x}}{\partial S_{i}}$ & $\frac{\partial \mu_{\mathrm{y}}}{\partial S_{i}}$ & $\frac{\partial \mu_{z}}{\partial S_{i}}$ & $\left|\frac{\partial \vec{\mu}}{\partial S_{i}}\right|$ & $\theta^{\mathrm{b}}$ \\
\hline (C) $\mathrm{CH}_{3}$ as & -0.449 & 0.049 & 0.0 & 0.452 & -6.2 \\
\hline (O) $\mathrm{CH}_{3}$ as & 0.400 & 0.225 & 0.0 & 0.459 & 29.3 \\
\hline (O) $\mathrm{CH}_{3} \mathrm{ss}$ & 0.468 & -0.433 & 0.0 & 0.637 & -42.8 \\
\hline (C) $\mathrm{CH}_{3} \mathrm{ss}$ & -0.116 & 0.283 & 0.0 & 0.306 & -67.8 \\
\hline $\mathrm{C}=\mathrm{Os}$ & -4.030 & -4.275 & 0.0 & 5.875 & 46.7 \\
\hline (O) $\mathrm{CH}_{3} \mathrm{ab}$ & -0.348 & -0.111 & 0.0 & 0.365 & 17.7 \\
\hline (C) $\mathrm{CH}_{3} \mathrm{ab}$ & 0.412 & 0.221 & 0.0 & 0.467 & 28.2 \\
\hline (O) $\mathrm{CH}_{3} \mathrm{sb}$ & -0.140 & -0.126 & 0.0 & 0.189 & 41.8 \\
\hline (C) $\mathrm{CH}_{3} \mathrm{sb}$ & -0.150 & 0.457 & 0.0 & 0.481 & -71.8 \\
\hline $\mathrm{C}_{2}-\mathrm{Os}$ & 5.110 & -0.765 & 0.0 & 5.167 & -8.5 \\
\hline (0) $\mathrm{CH}_{3}$ ip rock & 0.036 & 0.144 & 0.0 & 0.148 & 76.0 \\
\hline $\mathrm{CC} \mathrm{s}$ & 0.000 & 0.165 & 0.0 & 0.165 & 90.0 \\
\hline (C) $\mathrm{CH}_{3}$ ip rock & -0.261 & 0.049 & 0.0 & 0.266 & -10.6 \\
\hline $\mathrm{O}-\mathrm{Cs}$ & -2.260 & 3.145 & 0.0 & 3.873 & -54.3 \\
\hline $\mathrm{C}=\mathrm{O} \mathrm{ib}$ & 2.486 & -2.059 & 0.0 & 3.228 & -39.6 \\
\hline OCC d & 1.806 & -0.340 & 0.0 & 1.838 & -10.7 \\
\hline $\operatorname{COCd}$ & -2.728 & -0.292 & 0.0 & 2.744 & 6.1 \\
\hline (C) $\mathrm{CH}_{3}$ as & 0.0 & 0.0 & -0.350 & 0.350 & \\
\hline (O) $\mathrm{CH}_{3}$ as & 0.0 & 0.0 & -0.612 & 0.612 & \\
\hline (O) $\mathrm{CH}_{3} \mathrm{ab}$ & 0.0 & 0.0 & 0.379 & 0.379 & \\
\hline (C) $\mathrm{CH}_{3} \mathrm{ab}$ & 0.0 & 0.0 & 0.438 & 0.438 & \\
\hline (O) $\mathrm{CH}_{3}$ op rock & 0.0 & 0.0 & -0.017 & 0.017 & \\
\hline (C) $\mathrm{CH}_{3}$ op rock & 0.0 & 0.0 & -0.181 & 0.181 & \\
\hline $\mathrm{C}=\mathrm{O}$ ob & 0.0 & 0.0 & 0.460 & 0.460 & \\
\hline $\mathrm{C}_{2}-\mathrm{O}$ tor & 0.0 & 0.0 & -2.040 & 2.040 & \\
\hline $\mathrm{O}-\mathrm{C}$ tor & 0.0 & 0.0 & 0.008 & 0.008 & \\
\hline $\mathrm{CC}$ tor & 0.0 & 0.0 & -0.044 & 0.044 & \\
\hline
\end{tabular}

${ }^{a_{\mathrm{S}}}=$ stretch, as = antisymmetric stretch, $\mathrm{ss}=$ symmetric stretch, $\mathrm{ab}=$ antisymmetric bend, $\mathrm{sb}$ $=$ symmetric bend, $\mathrm{ib}=$ in-plane bend, $o b=$ out-of-plane bend, op = out-of-plane, $d=$ deformation, tor $=$ torsion.

${ }^{\mathrm{b}} \tan \theta=\left(\partial \mu_{y} / \partial S_{i}\right) /\left(\partial \mu_{x} / \partial S_{i}\right)$

$31 \mathrm{G}$ basis sets; the $6-31 \mathrm{G}$ values are given in Table 4 . Both sets of ab initio derivatives were used in the calculations of the IR band intensities. The eigenvector matrix $L$ in (1) was obtained from normal mode calculations using two different force fields: the force field from [8] (Set I) and the force field refined in this paper (Set II). Calculated IR band intensities are listed in Table 5 together with the experimental values. The absolute value of the IR intensity of the $\mathrm{C}=\mathrm{O}$ stretch normal mode (No. 5) was taken from ref. 15 . The relative IR intensities of the other modes listed in Table 5 were determined by numerical integration of the band areas in the spectrum of liquid $\mathrm{CH}_{3} \mathrm{COOCH}_{3}$ (the 
TABLE 5

Comparison of experimental and calculated IR band intensities of methyl acetate

\begin{tabular}{|c|c|c|c|c|c|}
\hline \multirow[t]{4}{*}{ Mode No. } & \multicolumn{5}{|c|}{ IR intensity $\left(\mathrm{km} \mathrm{mol}^{-1}\right)$} \\
\hline & \multirow[t]{3}{*}{ Experimental } & \multicolumn{4}{|c|}{ Calculated } \\
\hline & & \multicolumn{2}{|c|}{ Force field $I^{a}$} & \multicolumn{2}{|c|}{ Force field $\mathrm{II}^{\mathrm{b}}$} \\
\hline & & $3-21 \mathrm{G}$ & $6-31 G$ & $3-21 G$ & $6-31 G$ \\
\hline $1,2^{c}$ & 18.0 & 23.1 & 25.9 & 19.8 & 19.9 \\
\hline 3 & 35.8 & 23.7 & 25.0 & 28.1 & 30.5 \\
\hline 4 & 4.6 & 0.1 & 1.5 & 0.6 & 2.3 \\
\hline 5 & 281.3 & 236.0 & 279.9 & 248.7 & 311.7 \\
\hline $6,7,8,20,21^{d}$ & 97.6 & 55.1 & 63.0 & 67.6 & 76.3 \\
\hline 9 & 69.9 & 111.4 & 93.0 & 51.6 & 53.8 \\
\hline 10 & 355.8 & 325.5 & 323.4 & 430.3 & 405.9 \\
\hline 11 & $<3.0$ & 0.5 & 0.7 & 1.5 & 2.4 \\
\hline $12,23^{\mathrm{c}}$ & 112.3 & 85.4 & 76.2 & 62.8 & 69.2 \\
\hline 13 & 7.6 & 40.8 & 47.5 & 2.6 & 5.8 \\
\hline 14 & 38.2 & 31.5 & 36.1 & 33.7 & 38.3 \\
\hline 15 & 10.4 & 6.6 & 6.6 & 7.5 & 8.1 \\
\hline $18,19^{c}$ & 27.4 & 26.5 & 26.2 & 26.7 & 26.3 \\
\hline 22 & $<3.0$ & 1.6 & 2.5 & 1.7 & 2.5 \\
\hline 24 & 17.6 & 11.9 & 14.8 & 14.8 & 18.4 \\
\hline
\end{tabular}

${ }^{a}$ Force field from ref. $8 .{ }^{b}$ Force field from Table 2. ${ }^{\mathrm{c}}$ Two overlapping bands in IR spectrum. ${ }^{\mathrm{d}} \mathrm{Bands}$ corresponding to methyl bend modes except $(\mathrm{C}) \mathrm{CH}_{3} \mathrm{sb}$.

spectrum was measured on a Bomem DA3 FTIR spectrometer). Relative intensities were then normalized with respect to the published carbonyl band intensity [15]. Overlapping bands in the spectrum were separated by a leastsquares procedure using Lorentzian type functions as the band shapes. In cases of close overlap of bands, such that the separation of the bands could not be carried out reliably, the sum of the IR band intensities is given in Table 5. Only upper limits could be determined from the IR spectrum for the intensities of the very weak bands corresponding to normal modes No. 11 and No. 22 ( (O) $\mathrm{CH}_{3}$ in-plane and out-of-lane rock).

It can be seen from Table 5 that there is a good qualitative agreement between the experimental and calculated IR band intensities. This result supports the proposed assignments of the bands. A detailed comparison of the 3$21 \mathrm{G}$ and $6-31 \mathrm{G}$ results given in Table 5 indicates that the observed band intensities are better reproduced using the dipole derivatives calculated with the 6$31 \mathrm{G}$ basis, and this is true for both sets of force constants. In the amide group analysis [2] the best results were obtained using the $3-21 \mathrm{G}$ basis set.

Both force field sets I and II give fairly good agreement between observed 
and calculated frequencies of MA, with set II being somewhat better for the $\mathrm{CH}_{3}$ modes. From the point of view of predicted IR intensities (Table 5), the set I force field provides a better prediction of the absolute intensities of the two strongest bands (No. 5, C=O stretch, and No. 10, $\mathrm{C}_{2}-\mathrm{O}$ stretch), although not of their ratio, but it is somewhat poorer for the other bands. Its worst predictions, compared to set II, are for the weak bands, such as No. 4, No. 11, and particularly No. $13,(\mathrm{C}) \mathrm{CH}_{3}$ in-plane rock. These results demonstrate the very strong dependence of band intensities on the eigenvectors, and show that intensities can provide a sensitive test of a force field.

It is concluded that our force field and ab initio dipole derivatives provide a good reproduction of the observed vibrational frequencies and IR intensities of MA, and therefore can serve as satisfactory transferable parameters for the ester group.

\section{ACKNOWLEDGEMENTS}

This research was supported by S.C. Johnson and Son, Inc., and by the National Science Foundation (grants DMR-8303610 and DMB-8517812). We are indebted to T.C. Cheam for helpful discussions.

\section{REFERENCES}

1 T.C. Cheam and S. Krimm, Chem. Phys. Lett., 107 (1984) 613.

2 T.C. Cheam and S. Krimm, J. Chem. Phys, 82 (1985) 1631.

3 S. Krimm and Y. Abe, Proc. Nat. Acad. Sci. U.S.A., 69 (1972) 2788.

4 W.H. Moore and S. Krimm, Proc. Nat. Acad. Sci. U.S.A., 72 (1975) 4933.

5 J.K. Wilmshurst, J. Mol. Spectrosc., 1 (1957) 201.

6 Y.O. George, T.E. Houston and W.C. Harris, Spectrochim. Acta, Part A, 30 (1974) 1035.

7 P. Matzke, O. Chacon and C. Andrade, J. Mol. Struct., 9 (1971) 255.

8 H. Hollenstein and Hs.H. Günthard, J. Mol. Spectrosc., 84 (1980) 457.

9 V.M. Jardin-Barreto and J.J.C. Teixeira-Dias, Rev. Port. Quim., 23 (1981) 41.

10 C. Van Alsenoy, J.N. Scarsdale and L. Schäfer, J. Mol. Struct., 90 (1982) 297.

11 Y. Abe and S. Krimm, Biopolymers, 11 (1972) 1817.

12 A. Annamalai and S. Singh, Can. J. Chem., 61 (1983) 263.

13 H. Susi and J.R. Scherer, Spectrochim. Acta, Part A, 25 (1969) 1243.

14 C.M. Cook, Q.C.P.E., 13 (1981) 391.

15 W.A. Seth-Paul, Spectrochim. Acta, Part A, 30 (1974) 1817. 